\title{
IncRNA-ATB promotes stemness maintenance in colorectal cancer by regulating transcriptional activity of the $\beta$-catenin pathway
}

\author{
XIAOJIN YANG ${ }^{1,2^{*}}$, HANCHUAN TAO $^{1,2^{*}}$, CHENG WANG $^{3}$, WEIJUN CHEN $^{2}$, FU HUA $^{2}$ And HAIXIN QIAN ${ }^{1}$ \\ ${ }^{1}$ Department of General Surgery, First Affiliated Hospital of Soochow University, Soochow, Jiangsu 215006; Departments of \\ ${ }^{2}$ General Surgery and ${ }^{3}$ Neurology, Affiliated Dongtai Hospital of Nantong University, Dongtai, Jiangsu 224200, P.R. China
}

Received July 18, 2019; Accepted January 17, 2020

DOI: $10.3892 /$ etm.2020.8558

\begin{abstract}
Long non-coding RNA activated by transforming growth factor- $\beta$ (ATB) was recently reported to be involved in a wide range of physiological and pathological processes. However, the role of ATB in colorectal cancer (CRC) stemness remains unclear. In the present study, the functional role of ATB in maintaining stemness of CRC was determined using colony formation and sphere formation assays, and xenograft models. Reverse transcription-quantitative PCR, western blotting and immunohistochemistry were performed to investigate the mechanisms underlying the effects of ATB. Knockdown of ATB impaired colony formation and sphere formation in CRC cells, accompanied by an inhibition of colon tumor growth. Further results suggested that ATB regulated the transcriptional activity of the $\beta$-catenin pathway by inhibiting $\beta$-catenin expression. In addition, the results confirmed the role of $\beta$-catenin in ATB-mediated regulation of stemness in CRC. Collectively, the results indicated that ATB is a promising therapeutic target for CRC.

\section{Introduction}

Colorectal cancer (CRC) is a common clinical cancer that is associated with high morbidity and mortality (1). Gene mutations, inflammatory condition and imbalances in the microbiome are associated with increased risk of CRC development and progression (2-4). Despite advances in radiotherapy, chemotherapy and surgery, severe side effects compromise the CRC patient health $(5,6)$. Thus, there is an urgent interest and demand for identifying novel therapeutic targets and prognosis predictors for CRC.
\end{abstract}

Correspondence to: Dr Haixin Qian, Department of General Surgery, First Affiliated Hospital of Soochow University, 188 Shizi Street, Soochow, Jiangsu 215006, P.R. China

E-mail: haixinqiansoochow@163.com

*Contributed equally

Key words: colorectal cancer, long noncoding RNA activated by transforming growth factor- $\beta, \beta$-catenin
Long non-coding RNAs (lncRNAs) comprise a class of transcripts that are $>200$ nucleotides in length but without any protein-coding ability. Previous studies indicated that lncRNAs are involved in various physiological and pathological processes $(7,8)$. IncRNAs exert their biological functions by binding to DNA, RNA and proteins, thereby regulating protein expression at the transcriptional or post-transcriptional level $(9,10)$. Numerous lncRNAs are dysregulated in different types of cancer and are correlated with tumor cell proliferation, apoptosis, migration and stem-like properties (11-13). IncRNA activated by transforming growth factor- $\beta$ (ATB) has been identified as a novel transforming growth factor- $\beta$ (TGF- $\beta$ )-induced lncRNA that stimulates cancer cell proliferation, metastasis, epithelial-mesenchymal transition (EMT) and angiogenesis in lung, bladder, breast and gastric cancer (14-17). In CRC, ATB promoted cancer cell proliferation and was found to be a predictor of poor prognosis in patients $(18,19)$. However, the regulatory role of ATB in the maintenance of CRC cell stemness remains unclear.

The present study aimed to determine the functional role of ATB in maintaining the stemness of CRC cells. Colony formation assays, sphere formation assays and xenograft models were used to determine the functional role of ATB in maintaining CRC stemness. The mechanisms underlying the effects of ATB were additionally investigated by reverse transcription-quantitative PCR (RT-qPCR), western blotting, and immunohistochemical staining. The present study provided evidence for understanding ATB and identifying a potential therapeutic target for CRC.

\section{Materials and methods}

Cell culture and treatment. The human colon cancer cell lines HCT116 and HT29 were purchased from the American Type Culture Collection and cultured in DMEM containing 10\% FBS (Gibco; Thermo Fisher Scientific, Inc.). Lentivirus-based short hairpin RNAs (shRNAs) targeting ATB (shATB-1 and shATB-2, two different custom shRNA sequences targeting ATB) and shRNA-negative control (shNC) were obtained from Shanghai GenePharma Co., Ltd. HCT116 and HT29 cells were seeded into 6 well plates $\left(2 \times 10^{5}\right.$ cells per well). After culture for $24 \mathrm{~h}, 4 \times 10^{6}$ lentiviral particles were added into the well and puromycin $(2 \mathrm{mg} / \mathrm{ml})$ was added into the medium at $24 \mathrm{~h}$ post 
infection. The stably infected cells were collected for further experiments. A specific activator of the $\beta$-catenin pathway, CP21R7 (CP21; cat. no. S7954), was purchased from Selleck Chemicals and used to treat HCT116 and HT29 cells for $48 \mathrm{~h}$ at $37^{\circ} \mathrm{C}$ with a concentration of $2 \mathrm{mM}$. The equivalent volume of DMSO was added as a control.

Colony formation assay. A total of $2 \times 10^{3}$ HCT116 or HT29 cells were seeded into each well of a six-well plate containing $2 \mathrm{ml}$ DMEM supplemented with $10 \%$ FBS. A total of 14 days later, plates were collected and washed with PBS three times at room temperature. Next, $4 \%$ paraformaldehyde was added to fix the cells for $15 \mathrm{~min}$ at room temperature. After washing cells with PBS three times at room temperature, the cells were stained with $2 \%$ crystal violet (Beyotime Institute of Biotechnology) for $15 \mathrm{~min}$ at room temperature. The colonies in each well were counted under a light microscope (Nikon Corporation) by two experimenters and analyzed. Three independent experiments were performed in each group.

Sphere formation assay. A total of $2 \times 10^{4}$ HCT116 or HT29 cells were seeded into each well of a six-well plate containing $2 \mathrm{ml}$ serum-free DMEM. Five days later, the images of spheres were captured using an inverted microscope (x40; Nikon Corporation). The number of spheres in each well was counted after dilution for 100 times with the inverted microscope (x40; Nikon Corporation) by two experimenters and analyzed. Three independent experiments were performed in each group.

RNA extraction and RT-qPCR. Total RNA was extracted using TRIzol ${ }^{\mathrm{TM}}$ reagent (Invitrogen; Thermo Fisher Scientific, Inc.) according to the manufacturer's instructions. Reverse transcription (RT) was performed on isolated total RNA using a PrimeScript One Step RT-PCR kit (cat. no. RR047A; Takara Bio, Inc.), and qPCR was performed using a SYBR green Real Time PCR kit (Cat. No. RR085A; Takara Bio, Inc.), according to the manufacturer's protocol. The following thermocycling conditions were used for the qPCR: Initial denaturation at $95^{\circ} \mathrm{C}$ for $2 \mathrm{~min} ; 40$ cycles of $94^{\circ} \mathrm{C}$ for $15 \mathrm{sec}, 58^{\circ} \mathrm{C}$ for $30 \mathrm{sec}$ and $70^{\circ} \mathrm{C}$ for $30 \mathrm{sec}$; extension at $72^{\circ} \mathrm{C}$ for $5 \mathrm{~min}$. GAPDH was used as an internal control. The primers were supplied as follow: ATB-F, 5'-TCTGGCTGAGGCTGGTTGAC-3'; ATB-R, 5'-ATCTCTGGGTGCTGGTGAAGG-3'; GAPDH-F, 5'-TCAAGGCTGAGAACGGGAAG-3'; GAPDH-R, 5'-TCG CCCCACTTGATTTTGGA-3'. The relative gene expression was calculated using the $2^{-\Delta \Delta \mathrm{Cq}}$ method (20).

Western blotting. Total protein from HCT116 and HT29 cells was extracted using RIPA buffer (Beyotime Institute of Biotechnology) containing $1 \%$ protease inhibitor cocktail (EMD Millipore). The protein concentration was determined by BCA kit (Beyotime Institute of Biotechnology). Subsequently, $20 \mu \mathrm{g}$ of total protein was loaded in each lane of an SDS-PAGE (10\% for $\beta$-catenin, LEF1 and GSK-3 $\beta$ or $12 \%$ for GAPDH). Following electrophoresis, proteins were transferred onto PVDF membranes (EMD Millipore). Membranes were blocked with 5\% milk in TBS-Tween-20 buffer for $2 \mathrm{~h}$ at room temperature. The membranes were then incubated with primary antibodies supplied by Cell Signaling Technology, Inc. against $\beta$-catenin $(1: 1,000$; cat. no. 8480), lymphoid enhancer-binding factor 1 (LEF1; 1:80; cat. no. 76010) and glycogen synthase kinase-3 $\beta$ (GSK-3 $\beta ; 1: 1,000$; cat. no. 12456) overnight at $4^{\circ} \mathrm{C}$. Following incubation with HRP conjugated secondary antibody (1:10,000; cat. nos. ZDR 5306 and ZDR 5307; Origene Technologies, Inc.) at $22-25^{\circ} \mathrm{C}$ for $2 \mathrm{~h}$, an enhanced chemiluminescence kit (EMD Millipore) was used to detect specific bands. The density of each band was analyzed using ImageJ software (version: 1.52, National Institutes of Health).

Animal studies. Animal studies were performed in accordance with the institutional guidelines of Soochow University. Female BALB/c nude mice (age, 6 weeks; weight, 18-20 g; 5 mice in each group; humidity 40-60\%) were purchased from the Model Animal Research Center of Nanjing University and allowed to acclimate for 1 week before use. All of the mice were housed at $25^{\circ} \mathrm{C}$ under a $12 \mathrm{~h}$ light/dark cycle with ad libitum access to food and water. For xenograft models, 5x10 ${ }^{6}$ HT29 cells were injected into the right flank via the subcutaneous vein of mice. At 10 days post-injection, the tumor length and width was measured using a vernier caliper every 5 days. The tumor volume was calculated as tumor length $\mathrm{x}$ tumor width ${ }^{2}$ $\mathrm{x} 0.52$ (21). At 25 days post-injection, the mice were sacrificed and tumors were collected, weighed and used for histopathological studies.

Immunohistochemical staining and RNA-fluorescence in situ hybridization. The tumor tissues were fixed with $4 \%$ paraformaldehyde at $22-25^{\circ} \mathrm{C}$ for $48 \mathrm{~h}$ and embedded in paraffin. After heating at $65^{\circ} \mathrm{C}$ for $2 \mathrm{~h}$, the paraffin-embedded sections $(5 \mu \mathrm{m})$ of colon tumor tissues were dewaxed with xylene and gradient alcohol and then subjected to antigen retrieval with citrate buffer (OriGene Technologies, Inc.). Following blocking with $5 \%$ normal goat serum (Origene, Technologies, Inc.) at $37^{\circ} \mathrm{C}$ for $15 \mathrm{~min}$, the sections were incubated with primary antibody against $\beta$-catenin (1:200; cat. no. 8480, Cell Signaling Technology, Inc.) at $4{ }^{\circ} \mathrm{C}$ overnight. After immunoperoxidase staining with a Streptavidin-Peroxidase kit (OriGene Technologies, Inc.), 3,3'-diamino-benzidine (Fuzhou Maixin Biotechnology Development Co., Ltd.) was used to detect the target protein. The cell nucleus was stained with hematoxylin at $22-25^{\circ} \mathrm{C}$ for $3 \mathrm{~min}$ (Beyotime Institute of Biotechnology). For RNA-fluorescence in situ hybridization, a custom probe for the specific detection of ATB was synthesized by Guangzhou Ribobio Co., Ltd. The process of sample preparation and hybridization was followed by the use of a fluorescence in situ hybridization kit (cat. no. C10910; Guangzhou Ribobio Co., Ltd). Images of sections were captured by light microscope (x100; model BX51; Olympus Corporation). The $\beta$-catenin positive cells and total cells in each image were counted and the percent of positive cells were analyzed.

Statistical analysis. GraphPad Prism version 5.0 software (GraphPad Software, Inc.) was used for statistical analysis. One-way ANOVA followed with Dunnett's test was used to determine statistical significance for more than two groups. All data are presented as the mean \pm SEM. $\mathrm{P}<0.05$ indicated a statistically significant difference. 
A

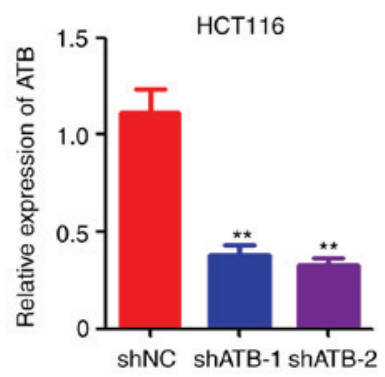

C

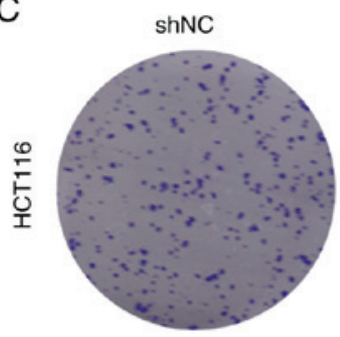

D

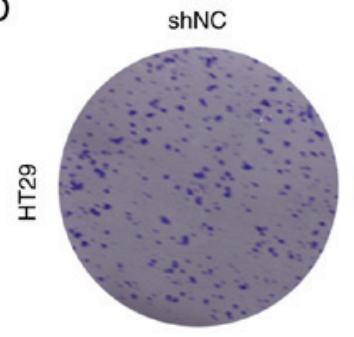

$E$

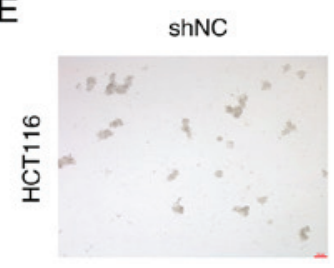

$\mathrm{F}$

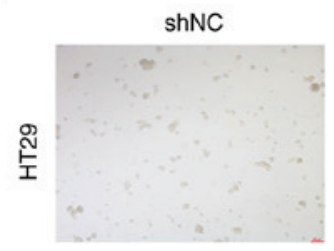

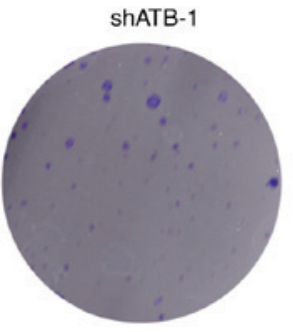

shATB-1

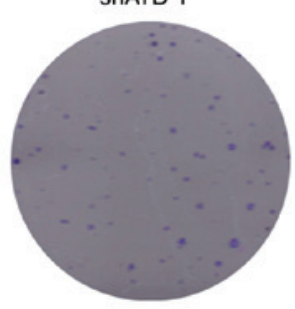

shATB-1

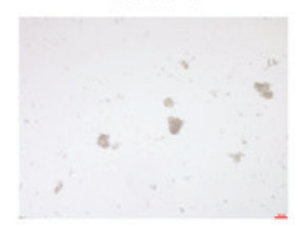

shATB-1

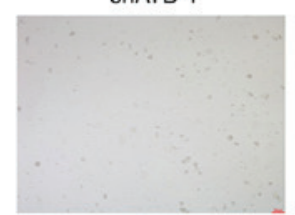

B

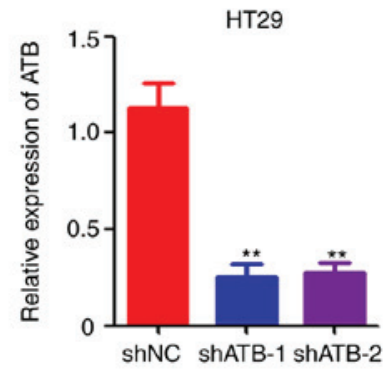

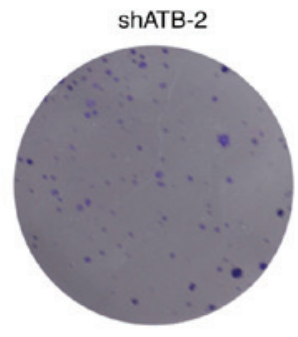

shATB-2

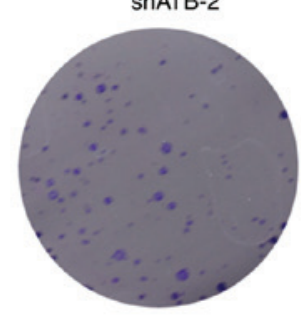

shATB-2

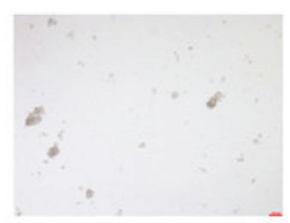

shATB-2

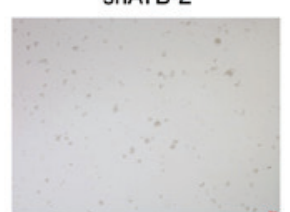

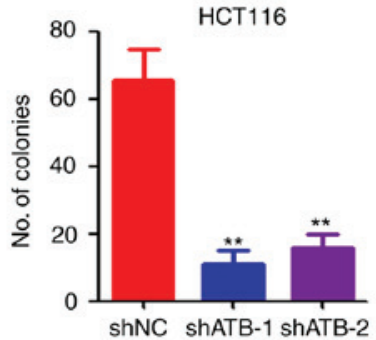

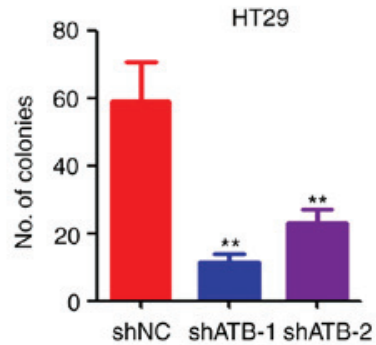

HCT116
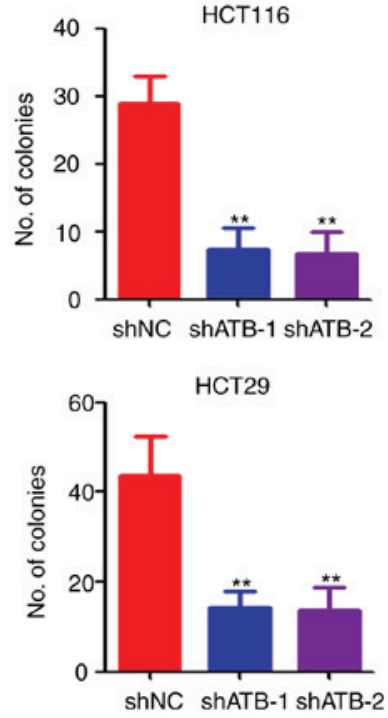

Figure 1. Knockdown of ATB impairs CRC stemness maintenance in vitro. Reverse transcription-quantitative PCR analysis of relative ATB expression in (A) HCT116 and (B) HT29 cells stably infected with shNC, shATB-1 and shATB-2. Colony formation assay of (C) HCT116 and (D) HT29 cells stably infected with shNC, shATB-1 and shATB-2. The number of colonies in each well were counted and analyzed (images were generated using a scanner without magnification). Sphere formation assay of (E) HCT116 and (F) HT29 cells stably infected with shNC, shATB-1 and shATB-2 (Scale bar=200 $\mu$ m). The number of colonies in each well was counted and analyzed. $n=3 .{ }^{* *} \mathrm{P}<0.01$ vs. NC. ATB, long non-coding RNA activated by transforming growth factor- $\beta$; sh, short hairpin RNA; NC, negative control.

\section{Results}

ATB knockdown impairs CRC stemness maintenance in vitro. To investigate the potential role of ATB in CRC, lentivirus-based shRNAs targeting ATB were used to infect HCT116 and HT29 cells. Following puromycin selection, stably-infected cells were collected for determination of ATB expression by RT-qPCR. The results indicated that shATB significantly downregulated ATB expression in both HCT116 (Fig. 1A) and HT29 (Fig. 1B) cells compared with shNC. The stably infected cells were then analyzed using a colony formation assay. Colony formation ability was significantly reduced in both HCT116 (Fig. 1C) and HT29 (Fig. 1D) cells after ATB knockdown. Furthermore, results of the sphere formation 

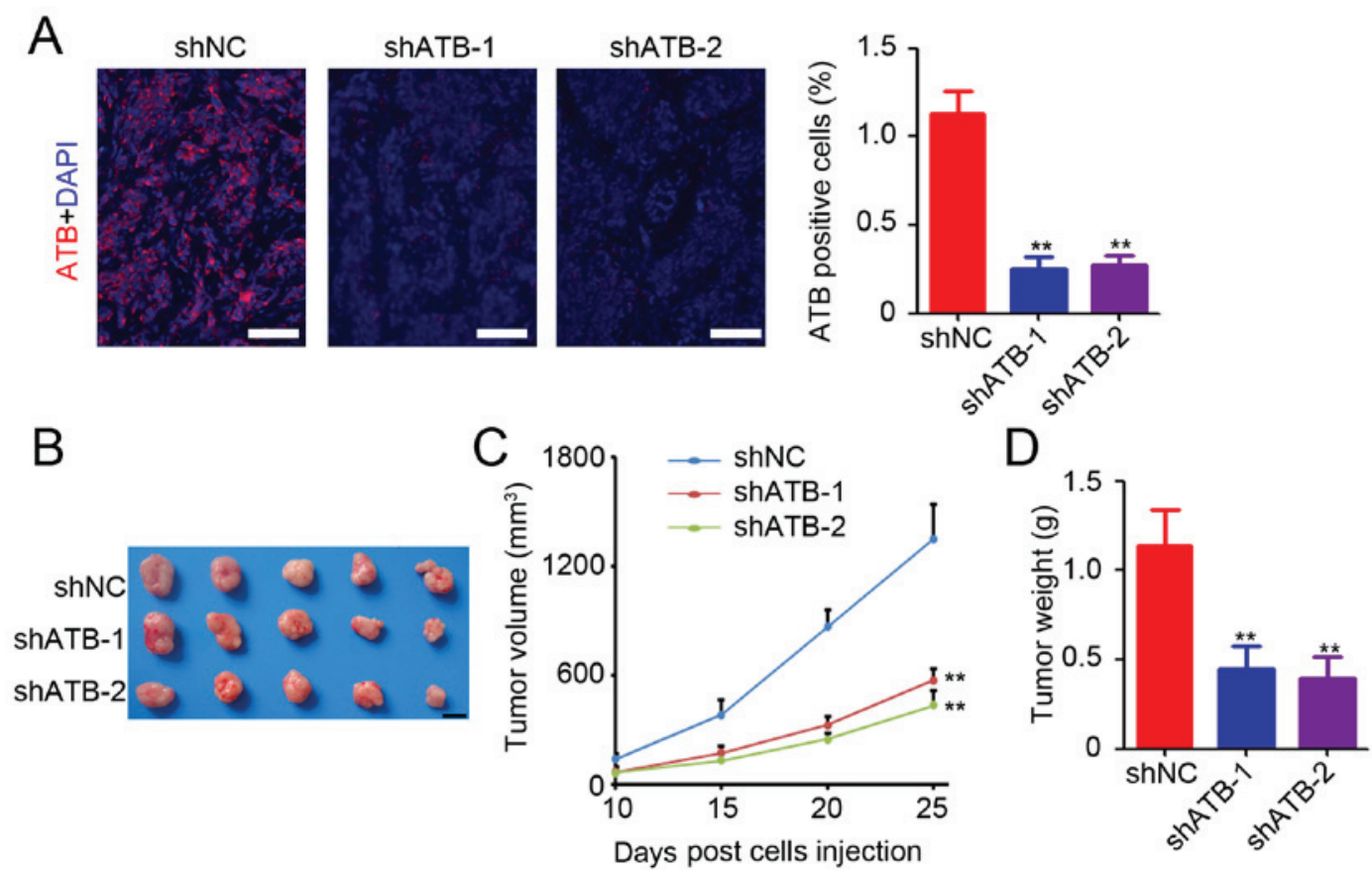

Figure 2. ATB promotes colon tumor growth in vivo. (A) Detection of ATB expression in HT29 tumor tissues by RNA-fluorescence in situ hybridization. Scale bar $=100 \mu \mathrm{m}$. The percentage of ATB-positive cells was then analyzed. $\mathrm{n}=3$. (B) Representative images of HT29 tumors from mice. Scale bar=1 cm. (C) The tumor volumes of HT29 tumors at 10, 15, 20 and 25 days post-injection. $n=5$. (D) The tumor weights of HT29 tumors. $n=5$. ${ }^{* * *} \mathrm{P}<0.01$ vs. shNC. ATB, long non-coding RNA activated by transforming growth factor- $\beta$; sh, short hairpin RNA; NC, negative control.

assay indicated that ATB knockdown significantly impaired sphere formation in HCT116 (Fig. 1E) and HT29 (Fig. 1F) cells. Collectively, the above results demonstrated the positive role of ATB in CRC stemness maintenance.

ATB promotes colon tumor growth in vivo. Based on the in vitro study results, an in vivo study was conducted to determine the functional role of ATB in colon tumor growth. HT29 cells that were stably transfected with shNC or shATB were injected into the right subcutaneous vein to establish a xenograft model. HT29 tumor tissues were collected for determination of ATB expression by RNA-fluorescence in situ hybridization. A small number of ATB-positive cells was observed in the colon tumor tissues after ATB knockdown (Fig. 2A). Further statistical analysis confirmed significant downregulation of ATB expression in shATB-infected HT29 tumors (Fig. 2A). The results suggested that ATB knockdown in HT29 cells significantly inhibited tumor growth (Fig. 2B), as evidenced by a mean reduction of $76 \%$ in tumor volume (shATB-1 and sh-ATB-2 vs. shNC; Fig. 2C) and 68\% decrease in tumor weight (shATB-1 and sh-ATB-2 vs. shNC; Fig. 2D). The above results demonstrated the oncogenic role of ATB in CRC.

$A T B$ inhibits the expression and activation of $\beta$-catenin . To determine the mechanisms underlying stemness maintenance in CRC by ATB, stably-transfected HCT116 and HT29 cells were collected for further molecular determination. Expression levels of the downstream targets of the Wnt signaling pathway were measured, and the results indicated that ATB knockdown significantly inhibited the expression of $\mathrm{G}_{1} / \mathrm{S}$-specific cyclin-D1, transcription factor-1, c-Myc, matrix metalloproteinase 7, and twist-related protein (Twist) both in HCT116 and HT29 cells compared with shNC (Fig. 3A). Western blotting was performed to determine the expression levels of $\beta$-catenin, LEF1, and GSK-3 $\beta$ in CRC cells. Significant downregulation of $\beta$-catenin expression was observed in both ATB-knockdown HCT116 and HT29 cells compared with shNC (Fig. 3B). However, ATB knockdown did not significantly affect the expression of LEF1 and GSK-3 $\beta$ in CRC cells (Fig. 3B). Reduced levels of $\beta$-catenin-positive cells were found in shATB-infected HT29 tumor tissues compared with shNC-infected HT29 tumor tissues (Fig. 3C). Taken together, the results demonstrated the regulatory role of ATB on $\beta$-catenin expression in CRC.

$\beta$-catenin plays a crucial role in ATB-mediated stemness maintenance in $C R C$. To determine whether $\beta$-catenin was important for regulating stemness in CRC by ATB, HT29 cells were treated with $\mathrm{CP} 21$, a specific activator of the $\beta$-catenin pathway. ATB knockdown significantly inhibited the colony formation ability of HT29 cells (Fig. 4A and B). However, CP21 treatment inhibited the decrease of colony formation mediated by shATB in HT29 cells (Fig. 4A and B). Collectively, the results confirmed that $\beta$-catenin was required for ATB-mediated maintenance of stemness in CRC.

\section{Discussion}

Considering their extensive biological functions, lncRNAs are involved in a wide range of physiological and pathological processes (22). In the present study, ATB knockdown impaired colony and sphere formation of CRC cells and significantly inhibited colon tumor growth. Further results suggested that 

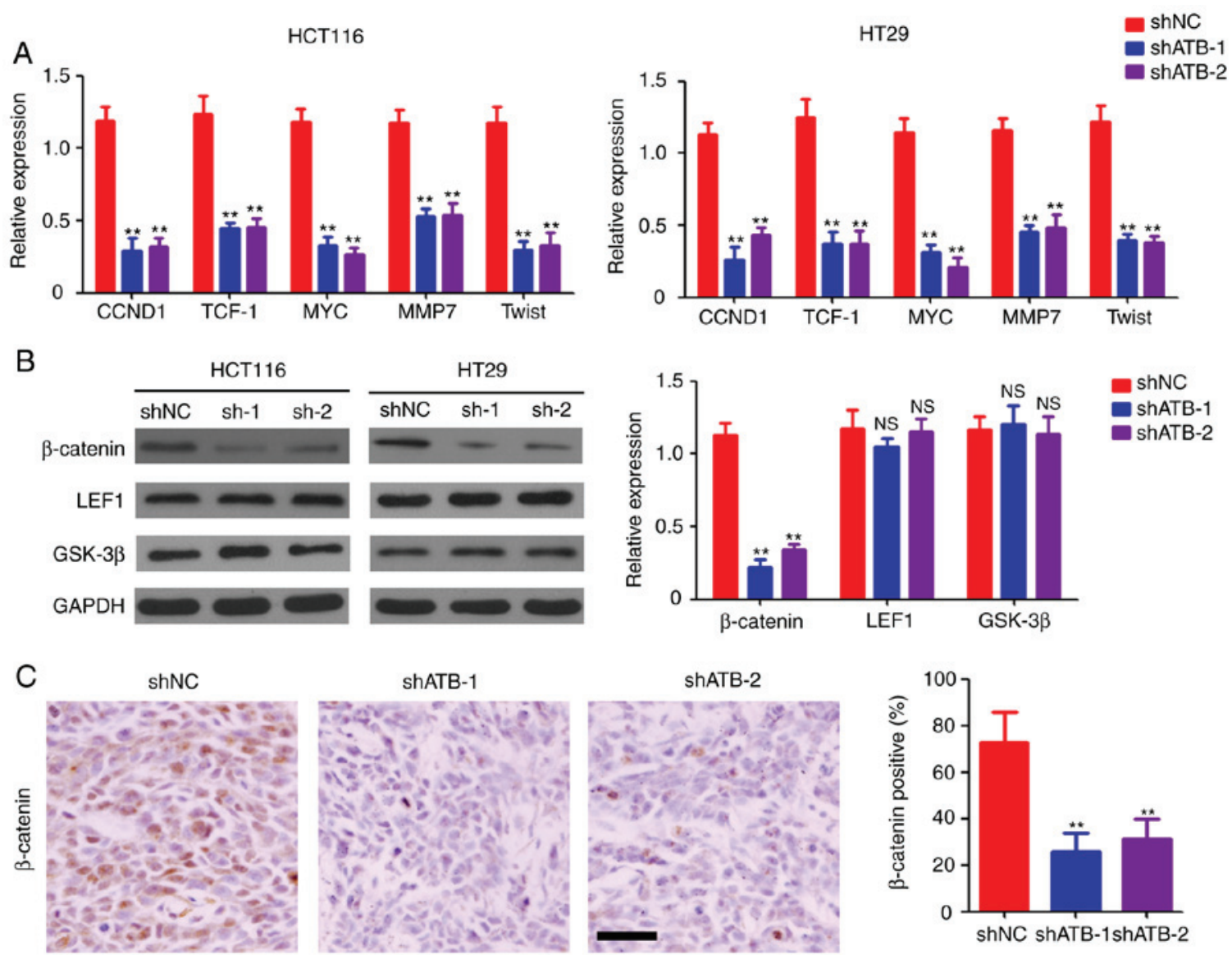

Figure 3. ATB regulates the activation of the Wnt/ $\beta$-catenin pathway. (A) Reverse transcription-quantitative PCR analysis of CCND1, TCF-1, MYC, MMP7 and Twist expression in HCT116 and HT29 cells stably transfected with shNC, shATB-1 and shATB-2. n=3. (B) Western blot analysis of $\beta$-catenin, LEF1 and GSK-3 $\beta$ expression in HCT116 and HT29 cells stably infected with shNC, shATB-1 and shATB-2. GAPDH was used as a loading control. The relative expression of each band was analyzed. $\mathrm{n}=3$. (C) Immunohistochemical staining of $\beta$-catenin expression in HT29 tumors (scale bar=100 $\mu \mathrm{m}$ ). The percentage of $\beta$-catenin-positive cells was analyzed. $\mathrm{n}=3 .{ }^{* *} \mathrm{P}<0.01$ vs. shNC. ATB, long non-coding RNA activated by transforming growth factor- $\beta$; sh, short hairpin RNA; NC, negative control; CCND1, cyclin-D1; TCF-1, transcription factor-1; MMP7, matrix metalloproteinase 7; Twist, twist-related protein; LEF1, lymphoid enhancer-binding factor 1 ; GSK-3 $\beta$, glycogen synthase kinase-3 $\beta$; NS, not significant.
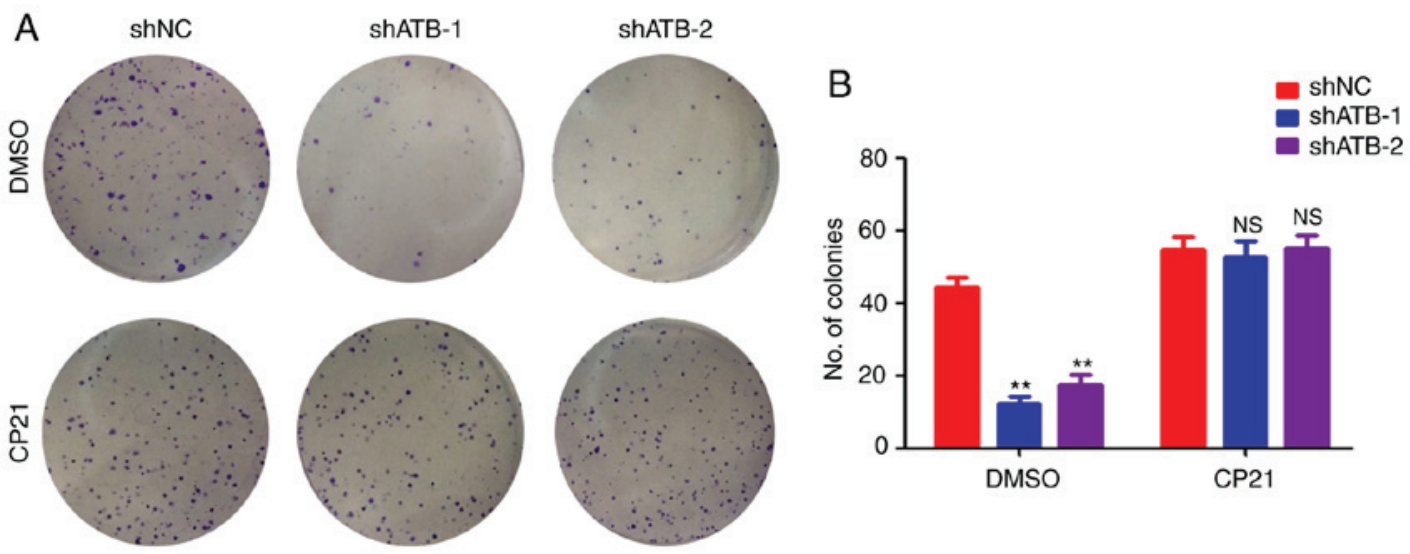

Figure 4. $\beta$-catenin plays a role in CRC stemness regulation by ATB. (A) Colony formation assay of HT29 cells that were stably transfected with shNC, shATB-1 and shATB-2 with or without CP21 treatment (images were generated using a scanner without magnification). (B) The number of colonies in each well was counted and analyzed. $n=3, \stackrel{*}{*} \mathrm{P}<0.01$ vs. shNC + DMSO group. ATB, long non-coding RNA activated by transforming growth factor- $\beta$; sh, short hairpin RNA; NC, negative control; NS, not significant.

ATB regulated the transcriptional activity of the $\beta$-catenin pathway by inhibiting $\beta$-catenin expression. In addition, the results confirmed that $\beta$-catenin was necessary for ATB-mediated regulation of stemness in CRC.
ATB is dysregulated in various types of cancer, including CRC (19), lung cancer (23), and liver cancer (24). ATB acts as an oncogene, and its dysregulation is commonly associated with poor prognosis of patients (25). In CRC, ATB is upregulated 
in colon cancer tissues and correlated with clinical cancer stage (19). In addition, ATB is highly expressed in lung cancer tissues and associated with tumor size and metastasis (23). A functional study indicated that ATB promoted proliferation, migration and invasion in osteosarcoma cells (26). Knockdown of ATB in endometrial cancer cells was found to impair cell viability by inducing caspase-3-related tumor apoptosis and $\mathrm{G}_{1} / \mathrm{S}$ arrest (27). In addition, ATB was responsible for the proliferation and apoptosis of CRC cells (18). The results demonstrated that ATB knockdown impaired stemness maintenance of CRC cells, indicated by the inhibition of colony formation and sphere formation, and colon tumor growth inhibition. The findings expand the current understanding of ATB function.

Recent studies demonstrated that ATB acts as a competing RNA by binding with microRNA (miR)-494 in lung cancer cells (28), miR-141-3p in gastric cancer cells (29), miR-590-5p in melanoma (30), and miR-200 family members in diverse types of cancer $(18,26,31,32)$. ATB regulates transcriptional coactivator YAP1 (YAP1), NF- $\mathrm{KB}, \mathrm{p} 38$ mitogen-activated protein kinase (MAPK), Twist, and TGF- $\beta$ in lung cancer, glioma, breast cancer and malignant melanoma $(23,30,33,34)$. In hepatic fibrosis, ATB knockdown downregulated $\beta$-catenin expression by upregulating the expression of endogenous miR-200a (35). In the present study, the results suggested that ATB regulated the transcriptional activity of the $\beta$-catenin pathway by inhibiting $\beta$-catenin expression. Further results confirmed the role of $\beta$-catenin during ATB-mediated regulation of stemness in CRC. miR-200a, which is involved in regulating CRC cell proliferation and $\beta$-catenin expression $(36,37)$, could serve as the direct target of ATB in regulating stemness maintenance. However, further studies are required to verify the above results.

The present study demonstrated the role of ATB in maintaining stemness of CRC by regulating $\beta$-catenin expression. However, further studies are required to investigate the direct target of ATB for regulating $\beta$-catenin expression in CRC. Collectively, the results indicated that ATB is a promising therapeutic target for CRC.

\section{Acknowledgements}

Not applicable.

\section{Funding}

No funding was received.

\section{Availability of data and materials}

The datasets used and/or analyzed during the current study are available from the corresponding author on reasonable request.

\section{Authors' contributions}

$\mathrm{XY}$ and HT were involved in the acquisition of the data and drafting the manuscript. $\mathrm{CW}, \mathrm{WC}$ and $\mathrm{FH}$ were involved in the analysis and interpretation of the data. HQ was involved in the conception and design of the present study. All authors read and approved the final manuscript.

\section{Ethics approval and consent to participate}

This animal study was approved by the Ethics Committee of Soochow University and complied with their guidelines.

\section{Patient consent for publication}

Not applicable.

\section{Competing interests}

The authors declare that they have no competing interests.

\section{References}

1. Bray F, Ferlay J, Soerjomataram I, Siegel RL, Torre LA and Jemal A: Global cancer statistics 2018: GLOBOCAN estimates of incidence and mortality worldwide for 36 cancers in 185 countries. CA Cancer J Clin 68: 394-424, 2018.

2. Dai L, Liu Y, Cheng L, Wang H, Lin Y, Shi G, Dong Z, Li J, Fan P, Wang Q, et al: SARI attenuates colon inflammation by promoting STAT1 degradation in intestinal epithelial cells. Mucosal Immunol 12: 1130-1140, 2019.

3. Brenner H, Kloor M and Pox CP: Colorectal cancer. Lancet 383: 1490-1502, 2014.

4. Humphries A and Wright NA: Colonic crypt organization and tumorigenesis. Nat Rev Cancer 8: 415-424, 2008.

5. Brungs D, Aghmesheh M, de Souza P, Carolan M, Clingan P, Rose J and Ranson M: Safety and efficacy of oxaliplatin doublet adjuvant chemotherapy in elderly patients with stage III colon cancer. Clin Colorectal Cancer 17: e549-e555, 2018.

6. Li J, Li XL, Yuan Y and Zhang SZ: Disputes and exploration of neoadjuvant and adjuvant therapy for colon cancer. Zhonghua Wei Chang Wai Ke Za Zhi 22: 329-335, 2019 (In Chinese).

7. Zheng J, Mao Y, Dong P, Huang Z and Yu F: Long noncoding RNA HOTTIP mediates SRF expression through sponging miR-150 in hepatic stellate cells. J Cell Mol Med 23: 1572-1580, 2019.

8. Wu S, Bono J and Tao YX: Long noncoding RNA (lncRNA): A target in neuropathic pain. Expert Opin Ther Targets 23: 15-20, 2019.

9. Liu H, Li C, Yang J, Sun Y, Zhang S, Yang J, Yang L, Wang Y and Jiao B: Long noncoding RNA CASC9/miR-519d/STAT3 positive feedback loop facilitate the glioma tumourigenesis. J Cell Mol Med 22: 6338-6344, 2018.

10. Yuan SX, Zhang J, Xu QG, Yang Y and Zhou WP: Long noncoding RNA, the methylation of genomic elements and their emerging crosstalk in hepatocellular carcinoma. Cancer Lett 379: 239-244, 2016.

11. Dai L, Li J, Dong Z, Liu Y, Chen Y, Chen N, Cheng L, Fang C, Wang $\mathrm{H}$, Ji Y, et al: Temporal expression and functional analysis of long non-coding RNAs in colorectal cancer initiation. J Cell Mol Med 23: 4127-4138, 2019.

12. Wu Q, Guo L, Jiang F, Li L, Li Z and Chen F: Analysis of the miRNA-mRNA-lncRNA networks in ER+ and ER-breast cancer cell lines. J Cell Mol Med 19: 2874-2887, 2015.

13. Lorenzi L, Avila Cobos F, Decock A, Everaert C, Helsmoortel H, Lefever S, Verboom K, Volders PJ, Speleman F, Vandesompele J and Mestdagh P: Long noncoding RNA expression profiling in cancer: Challenges and opportunities. Genes Chromosomes Cancer 58: 191-199, 2019.

14. Chen Y, Wei G, Xia H, Tang Q and Bi F: Long noncoding RNA-ATB promotes cell proliferation, migration and invasion in gastric cancer. Mol Med Rep 17: 1940-1946, 2018.

15. Xu S, Yi XM, Tang CP, Ge JP, Zhang ZY and Zhou WQ: Long non-coding RNA ATB promotes growth and epithelial-mesenchymal transition and predicts poor prognosis in human prostate carcinoma. Oncol Rep 36: 10-22, 2016.

16. Zhai $\mathrm{X}$ and $\mathrm{Xu}$ W: Long noncoding RNA ATB promotes proliferation, migration, and invasion in bladder cancer by suppressing MicroRNA-126. Oncol Res 26: 1063-1072, 2018.

17. Zhang Y, Li J, Jia S, Wang Y, Kang Y and Zhang W: Down-regulation of lncRNA-ATB inhibits epithelial-mesenchymal transition of breast cancer cells by increasing miR-141-3p expression. Biochem Cell Biol 97: 193-200, 2019. 
18. Gao Z, Zhou H, Wang Y, Chen J and Ou Y: Regulatory effects of lncRNA ATB targeting miR-200c on proliferation and apoptosis of colorectal cancer cells. J Cell Biochem 121: 332-343, 2020.

19. Yue B, Qiu S, Zhao S, Liu C, Zhang D, Yu F, Peng Z and Yan D: LncRNA-ATB mediated E-cadherin repression promotes the progression of colon cancer and predicts poor prognosis. J Gastroenterol Hepatol 31: 595-603, 2016.

20. Livak KJ and Schmittgen TD: Analysis of relative gene expression data using real-time quantitative PCR and the 2(-Delta Delta C(T)) method. Methods 25: 402-408, 2001

21. Cheng L, Yang Q, Li C, Dai L, Yang Y, Wang Q, Ding Y, Zhang J, Liu L, Zhang S, et al: DDA1, a novel oncogene, promotes lung cancer progression through regulation of cell cycle. J Cell Mol Med 21: 1532-1544, 2017

22. $\mathrm{Li} \mathrm{CH}$ and Chen $\mathrm{Y}$ : Insight into the role of long noncoding RNA in cancer development and progression. Int Rev Cell Mol Biol 326: 33-65, 2016.

23. Wei L, Wu T, He P, Zhang JL and Wu W: LncRNA ATB promotes the proliferation and metastasis of lung cancer via activation of the p38 signaling pathway. Oncol Lett 16: 3907-3912, 2018.

24. Lee YR, Kim G, Tak WY, Jang SY, Kweon YO, Park JG, Lee HW, Han YS, Chun JM, Park SY and Hur K: Circulating exosomal noncoding RNAs as prognostic biomarkers in human hepatocellular carcinoma. Int J Cancer 144: 1444-1452, 2019.

25. Li J, Li Z, Zheng W, Li X, Wang Z, Cui Y and Jiang X: LncRNA-ATB: An indispensable cancer-related long noncoding RNA. Cell Prolif: 50, 2017. doi: 10.1111/cpr.12381.

26. Han F, Wang C, Wang Y and Zhang L: Long noncoding RNA ATB promotes osteosarcoma cell proliferation, migration and invasion by suppressing miR-200s. Am J Cancer Res 7: 770-783, 2017.

27. Zheng X, Liu M, Song $Y$ and Feng C: Long noncoding RNA-ATB impairs the function of tumor suppressor miR-126-mediated signals in endometrial cancer for tumor growth and metastasis. Cancer Biother Radiopharm 34: 47-55, 2019.

28. Cao Y, Luo X, Ding X, Cui S and Guo C: LncRNA ATB promotes proliferation and metastasis in A549 cells by down-regulation of microRNA-494. J Cell Biochem 119: 6935-6942, 2018.

29. Lei K, Liang X, Gao Y, Xu B, Xu Y, Li Y, Tao Y, Shi W and Liu J: Lnc-ATB contributes to gastric cancer growth through a MiR-141-3p/TGF 32 feedback loop. Biochem Biophys Res Commun 484: 514-521, 2017
30. Mou K, Liu B, Ding M, Mu X, Han D, Zhou Y and Wang LJ: lncRNA-ATB functions as a competing endogenous RNA to promote YAP1 by sponging miR-590-5p in malignant melanoma. Int J Oncol 53: 1094-1104, 2018.

31. Li Z, Wu X, Gu L, Shen Q, Luo W, Deng C, Zhou Q, Chen X, Li Y, Lim Z, et al: Long non-coding RNA ATB promotes malignancy of esophageal squamous cell carcinoma by regulating miR-200b/Kindlin-2 axis. Cell Death Dis 8: e2888, 2017.

32. He T, Zhou H, Li C, Chen Y, Chen X, Li C, Mao J, Lyu J and Meng QH: Methylglyoxal suppresses human colon cancer cell lines and tumor growth in a mouse model by impairing glycolytic metabolism of cancer cells associated with down-regulation of c-Myc expression. Cancer Biol Ther 17: 955-965, 2016.

33. Zhou Y, Zheng X, Lu J, Chen W, Li X and Zhao L: Ginsenoside $20(\mathrm{~S})$-Rg3 inhibits the warburg effect via modulating DNMT3A/MiR-532-3p/HK2 pathway in ovarian cancer cells. Cell Physiol Biochem 45: 2548-2559, 2018.

34. Tang F, Wang H, Chen E, Bian E, Xu Y, Ji X, Yang Z, Hua X, Zhang $\mathrm{Y}$ and Zhao B: LncRNA-ATB promotes TGF- $\beta$-induced glioma cells invasion through NF- $\mathrm{B}$ and P38/MAPK pathway. J Cell Physiol 234: 23302-23314, 2019.

35. Fu N, Zhao SX, Kong LB, Du JH, Ren WG, Han F, Zhang QS, Li WC, Cui P, Wang RQ, et al: LncRNA-ATB/microRNA-200a/ $\beta$-catenin regulatory axis involved in the progression of HCV-related hepatic fibrosis. Gene 618: 1-7, 2017.

36. Santasusagna S, Moreno I, Navarro A, Martinez Rodenas F, Hernández R, Castellano JJ, Muñoz C and Monzo M: Prognostic impact of miR-200 family members in plasma and exosomes from tumor-draining versus peripheral veins of colon cancer patients. Oncology 95: 309-318, 2018.

37. Yang W, Ning N and Jin X: The lncRNA H19 promotes cell proliferation by competitively binding to miR-200a and derepressing $\beta$-catenin expression in colorectal cancer. Biomed Res Int 2017: 2767484, 2017.

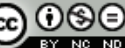

This work is licensed under a Creative Commons Attribution-NonCommercial-NoDerivatives 4.0 International (CC BY-NC-ND 4.0) License. 